\title{
O conceito de integralidade em saúde nos artigos científicos, no Supremo Tribunal Federal (STF) e nos enunciados do Conselho Nacional de Justiça (CNJ)
}

The health completeness concept in scientific articles, in the Federal Supreme Court (STF) and in the statements of the National Justice Council (CNJ)

El concepto de la integridad de la salud en artículos científicos, en el Supremo Tribunal Federal (STF) y en las declaraciones del Consejo Nacional de Justicia (CNJ)

Jéssica Resende Aguiar ${ }^{1}$

Alethele de Oliveira Santos ${ }^{2}$

RESUMO: Integralidade em saúde é considerada princípio de concepção polissêmica, entre as quais compreende a integração dos níveis de atenção, a articulação entre ações preventivas, curativas e de promoção. Sua compreensão é fundamental para o Sistema Único de Saúde (SUS) e para a execução de suas ações e serviços. Por meio de revisão bibliográfica, o artigo apresenta a compreensão desse princípio a partir de artigos científicos, decisão do Supremo Tribunal Federal (STF) e enunciados do Conselho Nacional de Justiça $(\mathrm{CNJ})$ e considera a necessidade de que haja parâmetros suficientes para sua prática diária.

Palavras-chave: Princípio da integralidade. Atenção integral à saúde.

ABSTRACT: Health integrity is considered a polissemic principle, among the integration of care levels, the relationship between preventive, curative and promotion of health actions. These comprehensions is fundamental to the Unified Health System (SUS) to the execution of their actions and services. Through a literature review, this essay presents the understanding of these principles of integrality throught scientific articles, Supreme Court (STF) decisions and recommendations of the National Council of Justice (CNJ) that considered the need of parameters for the daily practice.

Keywords: Principle of integrality. Primary health care.

RESUMEN: La completitud de la Salud tiene un deseño polisémico principio, que se incluyen la comprensión de niveles asistenciales, la prevención, recuperación y promoción de acciones de salud. Su comprensión es fundamental para el Sistema Único de Salud (SUS) y la ejecución de sus acciones y servicios. A través de la revisión de la literatura, este artículo presenta la comprensión del principio de la integralidad por exámenes de artículos científicos, decisión de la Corte Suprema (STF) y de publicaciones del Consejo Nacional de Justicia (CNJ) y considera una necesidad de suficientes parámetros para su práctica diaria.

Palabras-Ilave: Principio de integración. Atención integral de salud.

1 Especialista em Direito Sanitário pela Fundação Oswaldo Cruz - FIOCRUZ, Brasília, Brasil. jessicaaguiar@hotmail.com.

2 Alethele de Oliveira Santos, advogada, mestre em Saúde Coletiva pela UNB, Assessora Técnica do Conselho Nacional de Secretários de Saúde-CONASS, Brasília, Brasil. alethele@ig.com.br. 


\section{Introdução}

A ideia da integralidade foi expressa pela primeira vez no mundo, com a criação do National Health Service (NHS), no Reino Unido, em 1948, com a expansão das políticas sociais e dos sistemas de saúde. O NHS estabeleceu o princípio da responsabilidade coletiva por serviços completos e gratuitos para toda a população (1).

Passados 34 anos da difusão da ideia no mundo, a integralidade passou a fazer parte das propostas de reforma no Brasil, com a criação de programas para grupos específicos, como o Programa de Atenção Integral à Saúde da Criança (1).

Com a promulgação da Constituição Federal de 1988 (CF/88), o direito à saúde foi sagrado direito fundamental, sendo responsabilidade comum da União, dos Estados, dos Municípios e do Distrito Federal (art. 6º) (2). A Constituição e a Lei nº 8.080/90 delimitaram os princípios e diretrizes do Sistema Único de Saúde (SUS), sendo eles: a integralidade, a equidade, a descentralização, a participação social, a regionalização e a universalidade (3).

No artigo $7^{\circ}$, inciso II, da Lei $n^{\circ} 8.080 / 90$, a integralidade é conceituada: "integralidade de assistência, entendida como conjunto articulado e contínuo das ações e serviços preventivos e curativos, individuais e coletivos, exigidos para cada caso em todos os níveis de complexidade do sistema" (4).

A integralidade é considerada um dos princípios mais difíceis de alcançar e ao se consultar a literatura científica, percebe-se que tal princípio possui uma concepção polissêmica, "ora como eixo integrador de serviços, ora como visão holística do sujeito do cuidado ou como ações de atendimento integral de demandas e necessidades, entre outros" (5) (6).

Na Audiência Pública da Saúde, ocorrida em 2009, o representante do Conselho Nacional de Secretários Municipais de Saúde (Conasems), Antônio Carlos Figueiredo Nardi (7) aborda a necessidade de delimitação do conceito de integralidade:

\footnotetext{
É impossível e irracional se pensar que a integralidade seja um conceito aberto, solto no espaço, sem nenhuma regulamentação. A integralidade ilimitada, sem base científica, ética e orçamentária, e sem o compromisso com a coletividade não atende à própria Constituição, que impõe o dever de priorizar as ações e os serviços preventivos aos curativos. Além disso, é uma afronta ao bom senso e à hermenêutica do direito à saúde.
} 
Em resposta ao exposto, em 2011, com a promulgação da Lei no 12.401 que alterou a Lei nº 8.080/90, houve a regulação da assistência terapêutica e da incorporação de novas tecnologias. A lei estabeleceu que a dispensação de medicamentos deve seguir as diretrizes terapêuticas definidas no protocolo clínico para doenças ou agravos à saúde a ser tratado (art. 19-M) e na falta do protocolo, delimita três critérios para dispensação no art. 19-P (8).

Ao reafirmar que a integralidade é um dos princípios do Sistema Único de Saúde (SUS) que reúne vários significados, Assis - promotor de justiça do Estado de Minas Gerais - assevera que o princípio da integralidade é tridimensional (9). A primeira dimensão, extraída do art. 196 da CF/88, é considerada a dimensão horizontal, que referese "ao acesso universal e igualitário (leia-se equitativo) às ações e serviços para promoção, proteção e recuperação da saúde". A segunda dimensão é a integralidade sob a perspectiva vertical, que segue a parte final do art. 7, II, da Lei no 8.080/90. Tal dimensão pressupõe que o usuário tem por direito as ações e serviços de saúde nos diferentes graus de complexidade ou especialidade. Por fim, a última dimensão pressupõe um olhar humanizado e abordagem holística ao cuidado do indivíduo, se contrapondo ao cuidado fragmentado e com enfoque na doença (9).

Contudo, a integralidade, para o Poder Judiciário está relacionada à noção de consumo, a julgar pelo deferimento de demandas requerendo medicamentos e insumos da saúde sem levar em conta os Protocolos Clínicos e Diretrizes Terapêuticas (PCDT) relacionadas à doença. Tal viés restringe a saúde apenas à oferta de medicamentos e insumos (10).

Ao interpretar a integralidade como um princípio ilimitado, em que o Estado é obrigado a fornecer todos os insumos, medicamentos e tratamentos disponíveis no mercado, ocasiona, assim, um aumento de demandas judiciais (10). O atendimento dessas demandas colabora com o subfinanciamento da saúde. Ademais, a judicialização da saúde promove inequidades na saúde, pois:

Acaba privilegiando segmentos de doentes que têm mais recursos financeiros para pagar advogados, ou mais acesso à informação, em detrimento daqueles que deles têm mais necessidade (10).

Em contraponto à visão de que a integralidade deve ser interpretada como o 'tudo para todos', apresenta-se ponderação de que: 
A integralidade, corretamente interpretada nos sistemas de saúde, racionaliza a oferta de serviços e, por isso, transforma-se em instrumento fundamental de melhoria da eficiência dos gastos em saúde (11).

Demonstrada a inexistência de interpretação uníssona, a presente pesquisa mostrase tempestiva e adequada aos debates do SUS. Portanto, o presente trabalho tem como objetivos: descrever a produção científica sobre o princípio da integralidade do SUS, no período de 2010-2015, discutir o princípio a partir dos artigos científicos selecionados, da compreensão do Supremo Tribunal Federal (STF) na Suspensão de Tutela Antecipada 178 (12) e da compreensão do Conselho Nacional de Justiça (CNJ) nos enunciados 12, 14, 16 e 60 (13) (14).

\section{Metodologia}

Para dar conta do proposto, realizou-se pesquisa, a partir de revisão bibliográfica de artigos científicos publicados no período compreendido entre 2010 -2015, nas bases LILACS, IBECS, MEDLINE, Biblioteca Cochrane e SciELO, encontradas de forma compilada na Biblioteca Virtual em Saúde (BVS). A pesquisa foi realizada a partir de quatro descritores: "princípio da integralidade", "acesso integral à saúde e SUS", "acesso integral aos serviços de saúde" e "integralidade em saúde", utilizados isoladamente.

A partir dos filtros de pesquisa: texto completo, idioma português, artigos, anos 2010 a 2015, foram encontrados 189 artigos, sendo 64 para o descritor princípio da integralidade; 47 para acesso integral à saúde e SUS e 78 para acesso integral aos serviços de saúde. Dos 189 artigos, 32 eram duplicados e 31 artigos constaram em mais de um descritor. Por fim, resultaram 126 artigos.

Reunidos os artigos, foram utilizados como critério de exclusão: (i) os que se aplicavam à atuação de categorias profissionais; (i) os que se aplicavam a algum dos níveis de atenção; (iii) os que se aplicavam à integração entre níveis de atenção ou áreas da saúde; (iv) os que tratavam de formação profissional para a oferta de cuidados integrais à saúde; (v) os que tratavam da integralidade na atenção de uma população ou serviço específico.

A exclusão deu-se pela leitura e avaliação dos títulos e/ou resumos, permitindo delimitar a amostra em 36 artigos que tratavam do conceito do princípio da integralidade no SUS. 
Nos 36 artigos selecionados foi realizada leitura flutuante (15) e constatou-se que o conteúdo de 27 dos artigos referia-se à integralidade em uma parte da assistência, como exemplo, a integralidade em determinada unidade de atenção básica ou ainda, na atuação de categoria profissional da saúde, como a integralidade no atendimento dos profissionais da enfermagem.

Desta feita, restaram, conforme os objetivos do presente estudo, 9 arquivos a serem estudados.

A título de pesquisa complementar foram utilizados 4 artigos, dois publicados na coleção "Para entender a gestão no SUS" (16), livro "Direito à Saúde", do Conselho Nacional de Secretários de Saúde (Conass): (i) "O acesso à saúde pública e a eficácia das normas de regulação do SUS” (3), (ii) "Judicialização em saúde no estado de São Paulo" (17), o artigo publicado na revista Ciências \& Saúde Coletiva (iii) "Incorporação tecnológica no SUS: o problema e seus desafios" (18) e o artigo publicado na revista História, Ciência, Saúde-Manguinhos (iii) "Uma história de conceitos na saúde pública: integralidade, coordenação, descentralização, regionalização e universalidade" (19).

Todos os 13 arquivos selecionados foram lidos na íntegra e 9 deles sistematizados para agrupar suas principais características em tabelas comparativas. Os 4 artigos que não foram compilados na tabela, possuem uma conceituação diferenciada, por isso, não foram incluídos.

Foram ainda consideradas a decisão da Suspensão de Tutela Antecipada nำ178 do STF (16) e os enunciados 12, 14, 16 e 60 do CNJ (13) (14), vez que ambos os instrumentos têm o condão de orientar a intepretação do Poder Judiciário acerca do princípio da integralidade.

\section{Resultados e discussão}

Como forma didática de apresentação de resultados, optou-se por elaborar a tabela I, que apresenta os artigos selecionados em pesquisa na BVS, associados à literatura complementar e possibilita algumas análises imediatas. 
Tabela I: Artigos Selecionados para a pesquisa, por ano de publicação.

\begin{tabular}{|c|c|c|c|}
\hline $\begin{array}{c}\text { Ano de } \\
\text { publicação }\end{array}$ & Autor principal & Nome do artigo & $\begin{array}{l}\text { Número de } \\
\text { páginas }\end{array}$ \\
\hline 2015 & Siqueira & $\begin{array}{l}\text { Judicialização em saúde no estado de São Paulo } \\
\text { O acesso à saúde pública e a eficácia das normas }\end{array}$ & 15 \\
\hline 2015 & Dresch & $\begin{array}{l}\text { de regulação do SUS } \\
\text { Análise das demandas judiciais de medicamentos: } \\
\text { uma abordagem da realidade atual do distrito }\end{array}$ & 7 \\
\hline 2014 & Cardoso & $\begin{array}{l}\text { federal } \\
\text { Incorporação tecnológica no SUS: o problema e }\end{array}$ & 20 \\
\hline 2014 & Guimarães & $\begin{array}{l}\text { seus desafios } \\
\text { Os princípios da universalidade e integralidade do }\end{array}$ & 10 \\
\hline 2013 & Aith & $\begin{array}{l}\text { sus sob a perspectiva da política de doenças raras e } \\
\text { da incorporação tecnológica }\end{array}$ & 30 \\
\hline 2012 & Ayres & $\begin{array}{l}\text { Caminhos da integralidade: adolescentes e jovens } \\
\text { na Atenção Primária à Saúde } \\
\text { Direito à saúde e integralidade: uma discussão }\end{array}$ & 16 \\
\hline 2012 & Brito-Silva & $\begin{array}{l}\text { sobre os desafios e caminhos para sua efetivação } \\
\text { Estratégias para o Avanço da Integralidade na Visão }\end{array}$ & 12 \\
\hline 2012 & Higa & $\begin{array}{l}\text { de Professores e Estudantes } \\
\text { Integralidade na percepção dos trabalhadores de }\end{array}$ & 8 \\
\hline 2012 & Lima & $\begin{array}{l}\text { uma Unidade Básica de Saúde da Família } \\
\text { Uma história de conceitos na saúde pública: } \\
\text { integralidade, coordenação, descentralização, }\end{array}$ & 9 \\
\hline 2012 & Mello & $\begin{array}{l}\text { regionalização e universalidade } \\
\text { Equidade e inclusão de grupos sociais na política de }\end{array}$ & 21 \\
\hline 2011 & Albuquerque & $\begin{array}{l}\text { saúde: o caso do Recife, Brasil } \\
\text { Integralidade e Atenção Primária à Saúde: avaliação } \\
\text { na perspectiva dos usuários de unidades de saúde }\end{array}$ & 10 \\
\hline 2011 & Sala & $\begin{array}{l}\text { do município de São Paulo } \\
\text { Integração entre vigilância sanitária e assistência à } \\
\text { saúde da mulher: um estudo sobre a integralidade }\end{array}$ & 13 \\
\hline 2010 & Maia & no SUS & 11 \\
\hline Total & & & 182 \\
\hline
\end{tabular}

Fonte: Elaborada com base em consulta à BVS, 2010-2015.

Da tabela I, nota-se, de pronto, que tão logo publicada a Lei no 12.401 em 2011 (8), no ano subsequente houve um aumento de publicações de artigos relacionados ao tema. Porém, observado o conteúdo dos artigos publicados até o ano de 2012, a conceituação do princípio da integralidade permanece referenciada em autores anteriores a publicação da lei, permitindo inferir não ter havido inovação teórica.

É importante frisar que alguns autores consideram a integralidade um princípio polissêmico e, por isso, adotam ou consideram mais de uma conceituação para o tema ou, 
ainda, se valem de conceito amplo. Para Sala (20), "a integralidade pode ser entendida a partir de diversos ângulos não excludentes entre si, mas que destacam aspectos diversos da mesma questão".

A leitura dos artigos denotou, mesmo considerada a integralidade conceito polissêmico, a existência de abordagens comuns entre autores. A tabela II relaciona as abordagens apresentadas na conceituação de integralidade e os autores dos artigos utilizados. Para sua elaboração foram utilizados os significados apresentados por Albuquerque (21), efetuadas modificações de modo propiciar adequação aos demais autores:

a) integração das ações de promoção, proteção, recuperação e reabilitação, compondo níveis de atenção primária, secundária e terciária; b) forma de atuação profissional abrangendo as dimensões biológica, psicológicas e sociais; c) garantia de continuidade da atenção nos diversos níveis de complexidade do sistema, que demanda uma articulação entre serviços; d) conjunto de articulação de políticas públicas (21).

Quadro II - Relação entre as abordagens dos conceitos de integralidade e respectivos autores

Considera que o princípio da integralidade é:

Aith Albuquerque Ayres $\begin{gathered}\text { Brito- } \\ \text { Silva }\end{gathered}$ Higa Lima Maia Mello Sala $\begin{aligned} & \text { Total } \\ & (\%)\end{aligned}$

A articulação entre ações curativas, de prevenção de promoção.

$x \quad x$

$x \quad x \quad x \quad x$

$\mathrm{X} \quad \mathrm{X}$

89

Atendimento de todas as necessidades de saúde.

\begin{tabular}{|c|c|c|c|c|c|c|c|c|}
\hline & & $x$ & $x$ & $x$ & $x$ & & $x$ & 56 \\
\hline$x$ & & $x$ & & & & & & 22 \\
\hline & & & & $x$ & & & $x$ & 33 \\
\hline$X$ & & $x$ & $x$ & & & & & 33 \\
\hline$x$ & $x$ & $x$ & $x$ & $x$ & $x$ & $x$ & $x$ & 89 \\
\hline
\end{tabular}

Conjunto de articulação de políticas públicas.

Focada prioritariamente nas atividades preventivas e de promoção à saúde, $\quad X$ sem prejuízo dos serviços assistenciais.

Forma de atuação profissional abrangendo as dimensões biológica, psicológicas e sociais. Integração entre os níveis de atenção da saúde. Fonte:

Fonte: Elaborada com base em consulta à BVS, 2010-2015. 
À análise da tabela II considera-se que a compreensão do princípio da integralidade prioriza o acesso aos serviços e ações de saúde. Essa afirmativa decorre do elevado percentual de autores (aproximadamente $89 \%$ ) que defendem a integralidade conforme expressa no art. 7º, inciso II da Lei ํㅜ 8.080/90 (4), sendo a articulação entre as ações de prevenção, de promoção e curativas e como a integração dos níveis de atenção da saúde.

Nota-se, que mesmo após a publicação da Lei no 12.401/11, 3 dos 5 artigos publicados em 2012 permanecem com o entendimento de que o Estado brasileiro deve fornecer todas as necessidades de saúde.

Ayres et al (22), Brito-Silva (6), Higa et al (23), Lima et al (24), Maia et al (25) e Sala et al (20), com publicações entre 2010-2012, apresentam o princípio da integralidade segundo os escritos de Mattos $^{3}$, que indicam uma necessária definição e defesa de valores que the deem corpo e sua consequente prática.

Já os artigos publicados entre 2014 e 2015 apresentam a necessidade da regulamentação da integralidade, com foco, inclusive, na redução das demandas judiciais.

Aith et al (26), em seu trabalho, defende o conceito da integralidade como previsto no art. $7^{\circ}$, II, da Lei no ${ }^{\circ} .080 / 90$ (4), não excluindo os serviços de assistência farmacêutica e os de recuperação e reabilitação (21). Na mesma linha, Cardoso et al (27) defende a integralidade de acordo com a Lei ํo $8.080 / 90$ e o art. 198 da Constituição Federal de 1988.

Para Mello et al (18), o princípio da integralidade está relacionado à coordenação e visa promover a continuidade do cuidado e a integração entre ações preventivas e curativas.

Dos artigos cuja compilação foi expressa na tabela II, destaca-se Maia et al (25) que descreve os principais grupos de abordagens defendidos pela literatura: (i) medicina integral: defende a visão holística do ser humano e das práticas profissionais; (ii) integralidade nas políticas específicas: "são políticas direcionadas a populações ou portadores de doenças específicas, como idosos e portadores de HIV"; (iii) Integralidade na organização dos serviços: está relacionado à integração e coordenação entre os serviços e à continuidade da assistência. Sendo que, tal concepção polissêmica e conjugada é um

3 Mattos, RA: "Os sentidos da integralidade: algumas reflexões acerca de valores que merecem ser definidos"; "A integralidade na prática (ou sobre a prática da integralidade) "; e, "Os sentidos da integralidade: algumas reflexões acerca de valores que merecem ser defendidos", publicados em 2001, 2004 e 2006, respectivamente. 
desafio para o sistema de saúde, que adota os modelos "hospitalocêntricos", "medicalocêntrico", especializado e fragmentado.

Lima et al (24) também subdivide a integralidade em três atributos:

a) a integralidade enquanto atributo das práticas dos profissionais de saúde;

b) a integralidade na organização dos serviços de saúde, internamente, em cada serviço, articulando os diferentes níveis de atenção e entre os serviços diversos; c) a integralidade nas políticas especiais, apontada como a resposta governamental às necessidades de saúde específicas ou às necessidades de determinado grupo populacional, de modo a considerar seu contexto.

Essas autoras entendem, como atributo da prática profissional, que a atenção à saúde deve ser desenvolvida com um cuidado eficaz, humanizado e centrado nas necessidades do usuário individual ou coletivamente. Essa perspectiva considera o ser humano como todo, único e indivisível, superando, assim, o olhar fragmentado da saúde.

A integralidade relaciona-se às necessidades de saúde, classificando-as em quatro conjuntos: ter boas condições de vida; ter acesso e possibilidade de consumo de todas as tecnologias de saúde; criar vínculos efetivos com o profissional e/ou equipe; e ter autonomia no seu modo de andar a vida. Nessa perspectiva, as práticas dos profissionais e das equipes que se pautam pela integralidade remetem a um olhar sobre o sujeito, o significado que atribui ao cuidado e a sua autonomia no modo de ser e levar a vida (24).

Brito-Silva (6), para quem a Constituição relacionou o conceito de integralidade com a lógica do acesso e integração dos níveis de atenção. Ele apresenta em seu artigo um conceito de integralidade que compreende desde aspectos relacionados à regulação política do setor saúde, até à lógica de atuação de equipe multidisciplinar, que ofereça um cuidado que envolva todas as dimensões do ser humano.

Essas especificações estão relacionadas com os $22 \%$ dos autores compreendem a integralidade como um conjunto de articulação de políticas públicas. Tal viés da integralidade é de importância, já que compreende as respostas governamentais dadas aos problemas específicos de saúde (6). É nessa compreensão da integralidade que se incluem as políticas direcionadas às necessidades da população, como exemplo, a Política Nacional de Atenção Integral à População Negra ou a Política Nacional de Atenção Integral às Pessoas com Doenças Raras.

Para Higa et al (23) a integralidade é um dos princípios do SUS menos evidenciado. Para ela, a integralidade é dividida em três sentidos. O primeiro sentido emprega às 
politicas e ações governamentais, promovendo a prevenção e atenção das necessidades da população. O segundo sentido se associa à organização dos serviços de saúde. E, por fim, o último sentido é voltado aos atributos da prática da saúde.

Ayres et al (22) adotam uma definição de integralidade mais abrangente, dividindo em quatro eixos que abordam, segundo os autores, os aspectos mais relevantes:

A) eixo das necessidades: diz respeito à qualidade e natureza da escuta, acolhimento e resposta às demandas de atenção à saúde; o impulso mais relevante aqui é o do desenvolvimento de sensibilidade e capacidade de resposta a necessidades que não se restrinjam à prevenção, correção e recuperação de distúrbios morfológicos ou funcionais do organismo, sem descuidar destas;

B) eixo das finalidades: diz respeito a graus e modos de integração entre as ações de promoção da saúde, prevenção de agravos, tratamento de doenças e sofrimentos, e recuperação da saúde/ reinserção social; o sentido dominante aqui é o de não segmentar as ações, mas, ao contrário, criar sinergismos que otimizem o desenvolvimento das ações, tanto da perspectiva da racionalização meios-fins, como do conforto e conveniência para indivíduos, famílias e comunidades;

C) eixo das articulações: refere-se aos graus e modos de composição de saberes interdisciplinares, equipes multiprofissionais e ações intersetoriais no desenvolvimento das ações e estratégias de atenção à saúde; o propósito aqui é criar as melhores condições para oferecer resposta efetiva às necessidades de saúde em uma perspectiva ampliada, tal como descrito no item A;

D) eixo das interações: refere-se à qualidade e natureza das interações intersubjetivas no cotidiano das práticas de cuidado; a motivação das propostas identificadas nesse eixo é a construção de condições efetivamente dialógicas entre os sujeitos participantes dos encontros relacionados à atenção à saúde, sejam de pessoa a pessoa, sejam na perspectiva de equipes/comunidades, sem o que as aspirações dos eixos anteriores não podem ser realizadas (22).

Ainda em relação à tabela II, observa-se que aproximadamente $56 \%$ dos autores defendem que a compreensão é de que o princípio estudado, expressa a obrigatoriedade do Estado em fornecer atendimento integral à população. Nesse contexto, cabe apresentar Dresch (3) que indica a parte da doutrina para quem o direito à saúde se enquadra no mínimo existencial, ou seja, o Estado só tem a obrigação de fornecer um conjunto limitado de ações que garantam a dignidade humana.

Cardoso (27), Dresch (3), Guimarães (18) e Siqueira (17), apresentaram conceitos exclusivos, que não encontraram guarida na abordagem selecionada, e, portanto, não foram expressos no Quadro II. 
Siqueira (17) afirma que o princípio da integralidade não pode ser interpretado considerando apenas as normas constitucionais, pois a análise isolada das normas induz a uma interpretação equivocada de que o Estado deva oferecer, em qualquer situação, o desejado para manutenção ou recuperação da saúde individual. Nessa compreensão “isolada”, são desconsideradas as politicas públicas e os PCDT do SUS.

Para as necessidades de saúde, deve-se levar em conta os regulamentos técnicos e científicos, protocolos de conduta, limites para incorporação de tecnologias, protocolos farmacêuticos, os estudos científicos de custo-benefício, de eficácia, efetividade e segurança. Sob tal ótica, a integralidade não admite toda e qualquer terapêutica existente (3) (17).

Aith et al (26) e Guimarães (18) também defendem em seus textos que a interpretação equivocada do princípio ocasiona aumento de ações judiciais requerendo tratamentos e insumos da saúde, o que traz como consequência o crescimento dos gastos com as ações judiciais. Tal ponto de vista é perceptível no estudo realizado por Cardoso (27) que mostra que de 246 medicamentos requeridos pela via judicial, apenas $21,5 \%$ deles constavam na Relação Nacional dos Medicamentos Essenciais (RENAME).

Mesmo após a aprovação da Lei n. 12.401/11 (8), do Decreto n. 7.508/12 (28) e do Decreto n. 7.646/11 (29), que delimitam os contornos da assistência farmacêutica e da incorporação de tecnologia no SUS e, ainda, após o julgamento da STA nำ178, que privilegia o tratamento previsto pelo SUS em detrimento de opção diversa pretendida pelo paciente ou profissional da saúde, "a questão do que é ou não devido pelo SUS ainda não está encerrada e a judicialização da saúde no SUS continua crescente" (18).

Pode-se crer que as diversas compreensões do princípio da integralidade acabam por fomentar a judicialização da saúde, principalmente quando interpretada que o Estado deve fornecer todas as expectativas em saúde. Ainda que a atuação do Poder Judiciário seja imprescindível para a efetivação da cidadania e do direito à saúde, é necessário que haja uma redução de demandas judiciais ou uma limitação do fornecimento de tratamentos e medicamentos experimentais (12).

Contudo, na mesma decisão, o STF, indica a possibilidade de que haja solicitação de procedimento, tratamento ou medicamento não previsto pelas políticas do SUS, denotando uma análise caso a caso, inclusive pela possibilidade de que o item existente nos PCDT não possua eficácia para o paciente (12). 
Observa-se, que há diferenças no entendimento do STF e dos artigos estudados. O STF, na STA n. 178 (12), indica uma ponderação entre regras existentes e a aplicabilidade delas ao caso concreto, os artigos estudados, em maioria, oferecem conceitos mais amplos, à exceção de 4 autores que defendem alguma limitação.

Com o intuito de orientar a interpretação das leis pelos juízes em julgamentos da área da saúde e colaborar com a atuação do judiciário (30), o CNJ produziu uma série de enunciados interpretativos sobre o direito à saúde, durante as I e II Jornadas de Direito da Saúde (13) (14). Cabe ressaltar que "o objetivo dos enunciados de saúde, assim como já ocorreram em outros temas, é orientar e tentar encontrar soluções harmonizadoras procedimentais para as demandas que sobrecarregam o judiciário" (28).

Baseando-se nesse entendimento, o enunciado oㅜ 12, segue a mesma linha de compreensão do STF, evidenciando a "compreensão subjetiva" da integralidade, a fim de ser analisado no caso concreto (13).

Segundo o enunciado ํo 16 (13), ao requerer um tratamento ou medicamento não previsto pelos protocolos, o autor do requerimento deve apresentar evidências científicas que comprovem a "inexistência, inefetividade ou impropriedade dos procedimentos ou medicamentos constantes dos protocolos clínicos do SUS". Caso não seja comprovada a "ineficiência ou impropriedade dos medicamentos e tratamentos" oferecidos pelo SUS, o enunciado ํo 14 determina que deve ser negado o pedido, caso não conste nas políticas de saúde (13).

Do enunciado 60 é possível verificar o que vem se chamando de compreensão subjetiva do princípio da integralidade:

Protocolos Clínicos e Diretrizes Terapêuticas (PCDT) são elementos organizadores da prestação farmacêuticas, de insumos e de procedimentos, e não limitadores. Assim, no caso concreto, quando todas as alternativas terapêuticas previstas no respectivo PCDT já tiverem sido esgotadas ou forem inviáveis ao quadro clínico do paciente usuário do SUS, pelo princípio do art. 198, II, da CF, pode ser determinado judicialmente o fornecimento, pelo Sistema Único de Saúde, do fármaco, insumo ou procedimento não protocolizado (14).

Tal compreensão diverge do que foi exposto por Dresch (3) e Siqueira (17), que defendem que a integralidade deve-se basear nos regulamentos e protocolos previstos. 
Os enunciados 4, 5, 6, 9, 50, 57, 58 e 59 (13) (14), em alguma medida também impõem limitações aos itens de saúde, por ventura, pretendidos judicialmente e reiteram a ponderação anteriormente exposta.

\section{Considerações finais}

Nota-se que até 2012 há uma polissemia de definições para o princípio em estudo, sendo que em mais da metade dos artigos analisados a compreensão é de que a integralidade é, necessariamente, o fornecimento de todas as necessidades de saúde.

Considera-se que houve uma reavaliação nos conceitos após a publicação da Lei no 12.401/11. Somente artigos publicados depois de 2014 trazem a compreensão de que a integralidade não pode ser entendida de forma ampla, e defendem a necessidade da regulação de acordo com os protocolos técnicos definidos pelo SUS. Essa compreensão, ainda que de alguma forma tenha sido reproduzida pelo STF e pelo CNJ, não foi suficiente para regrar a análise dos casos concretos, quando nos processos judiciais.

É de se considerar, em que pese todos os estudos elaborados, e as posições já tomadas pelo STF e pelo $\mathrm{CNJ}$, essas se revelam insuficientes para a prática diária do princípio da integralidade, que requer definições e parâmetros explícitos, minimamente, sob pena de ver crescer as ações judiciais que versam sobre o direito à saúde.

\section{Referências}

1 Conill EM. Avaliação da integralidade: conferindo sentido para os pactos na programação de metas dos sistemas municipais de saúde. Cad. Saúde Pública. 20(5): 1417-1423.

2 Brasil. Constituição Federal de 1988. Brasília, DF. Disponível em: http://www.planalto.gov.br/ccivil_03/constituicao/ConstituicaoCompilado.htm. [Acesso em 2015, 14.set].

3 Dresch RL. O acesso à saúde pública e a eficácia das normas de regulação do SUS. Coleção para entender a gestão do SUS. Conselho Nacional de Secretários de Saúde. Brasília: CONASS, 2015.

4 Brasil. Lei o 8.080, de 19 de setembro de 1990. Brasília, DF. Disponível em: http://www.planalto.gov.br/ccivil_03/Leis/L8080.htm. [Acesso em 2015, 14.set].

5 Silva RVGO, Ramos FRS. Integralidade em saúde: revisão de literatura. Cienc Cuid Saude $2010 \mathrm{Jul} / \mathrm{Set}$; 9(3):593-601. 
6 Brito-Silva, K, Bezerra, AFB, Tanaka, OY. Direito à saúde e integralidade: uma discussão sobre os desafios e caminhos para sua efetivação. Interface. 2012, 16 (40): 249-260.

7 Santos, AO. Discursos proferidos na audiência pública da saúde do Supremo Tribunal Federal: uma análise à luz da teoria dos sistemas sociais. 2013. 353, 292 f., il. Dissertação (Mestrado em Saúde Coletiva) - Universidade de Brasília, Brasília, 2013.

8 Brasil, Lei no 12.401, de 28 de abril de 2011. Disponível em:

http://www.planalto.gov.br/ccivil_03/_Ato2011-2014/2011/Lei/L12401.htm. [Acesso 01.nov 2015]

9 Oliveira, LM. Garantia do acesso integral à saúde na atenção oncológica. Disponível em: https://aplicacao.mpmg.mp.br/xmlui/bitstream/handle/123456789/994/R\%20DJ\%20Coment \%C3\%A1 rio\%20jurisp\%20garantia\%20acesso\%20-

\%20luciano\%20moreira\%20\%5b\%E2\%80\%A6.pdf?sequence=1. [Acesso em 02 nov.2015].

10 Vieira FS. Ações judiciais e direito à saúde: reflexão sobre a observância aos princípios do SUS. Rev. Saúde Pública. 2008. 42(2): 365-369.

11 Brasil. Sistema Único de Saúde. Coleção Progestores - Para entender a gestão do SUS, 1. Conselho Nacional de Secretários de Saúde - Brasília: CONASS, 2007.

12 Brasil. Suspensão de tutela antecipada no 178. Disponível em: http://www.stf.jus.br/portal/processo/verProcessoTexto.asp?id=2369969\&tipoApp=RTF. [Acesso em 2 nov 2015].

13 Conselho Nacional de Justiça. Enunciados aprovados na I jornada de direito da saúde do conselho nacional de justiça em 15 de maio de 2014 - São Paulo/SP. Disponível em: http://www.defensoria.ba.gov.br/portal/arquivos/downloads/ENUNCIADOS_APROVADOS NA_JORNADA_DE_DIREITO_DA_SAUDE_PLENARIA_15_5_14.pdf. [Acesso em $02 \mathrm{nov}$ 2015].

14 Conselho Nacional de Justiça. Enunciados aprovados II jornada de direito da saúde. Disponível em:

http://www.cnj.jus.br/files/conteudo/destaques/arquivo/2015/05/96b5b10aec7e5954fcc1978 473e4cd80.pdf. [Acesso em 2 nov 2015.].

15 Minayo, MSC. O desafio do conhecimento. Pesquisa qualitativa em saúde. $8^{a}$ ed. São Paulo: HUCITEC; 2007.

16 Brasil. Direito à saúde. Coleção para entender a gestão do SUS. Conselho Nacional de Saúde- Brasília: CONASS, 2015.

17 Siqueira PSF. Judicialização em saúde no estado de São Paulo. Coleção para entender a gestão do SUS. Conselho Nacional de Secretários de Saúde. - Brasília: CONASS, 2015.

18 Guimarães R. Incorporação tecnológica no SUS: o problema e seus desafios. Ciência \& Saúde Coletiva, 19 (12):4899-4908, 2014. 
19 Mello, GA, Viana, ALA. Uma história de conceitos na saúde pública: integralidade, coordenação, descentralização, regionalização e universalidade. Hist. cienc. saudeManguinhos. 19 (4): 1219-1239.

20 Sala $A$, Luppi CG, Simões $O$, Marsiglia RG. Integralidade e atenção primária à saúde: avaliação na perspectiva dos usuários de unidades de saúde do município de São Paulo. Saúde Soc. São Paulo, 2011, 20 (4): 948-960.

21 Albuquerque, MSV, Costa AM, Lima LP, Melo FDA de. Equidade e inclusão de grupos sociais na política de saúde: o caso do Recife, Brasil. Rev. Bras. Saude Mater. Infant. 11 (1): 73-82.

22 Ayres, JRCM, Carvalho YM de, Nasser MA, Saltão RM, Mendes VM. Ways of comprehensiveness: adolescentes and young adults in primary. Healthcare. Interface Comunic., Saude, Educ., 16 (40): 67-81, jan./mar. 2012.

23 Higa, EFR, Yamada DM, Peloso GFB, Peres CRFB, Panacionne SZE, Mesquita CMB. Estratégias para o avanço da integralidade na visão de professores e estudantes. Rev. bras. educ. med.2012, 36 (4): 470-477.

24 Lima, IFS, Lobo FS, Acioli KLBO, Aguiar ZN. Integralidade na percepção dos trabalhadores de uma Unidade Básica de Saúde da Família. Rev. esc. enferm. USP 2012, 46 (4): 944-952.

25 Maia, C, Guilhem D, Lucchese G. Integração entre vigilância sanitária e assistência à saúde da mulher: um estudo sobre a integralidade no SUS. Cad. Saúde Pública. 2010, 26 (4): 682-692.

26 Aith, F, Bujdoso Y, NPR, Dallari SG. Os princípios da universalidade e integralidade do SUS sob a perspectiva da política de doenças raras e da incorporação tecnológica. Revista de Direito Sanitário, Brasil, 2015,15 (1): 10-39.

27 Cardoso, CS. Análise das demandas judiciais de medicamentos: uma abordagem da realidade atual do distrito federal. Revista Jurídica da Procuradoria-Geral do Distrito Federal, Brasília, 2014, 39 (1): 11-30.

28 Brasil. Decreto o 7.508, de 28 de junho de 2011. Disponível em http://www.planalto.gov.br/ccivil_03/_ato2011-2014/2011/decreto/D7508.htm. [Acesso 2015, 16 nov.].

29 Brasil. Decreto ํo 7.646, de 21 de dezembro de 2011. Disponível em http://www.planalto.gov.br/ccivil_03/_Ato2011-2014/2011/Decreto/D7646.htm. [Acesso em 16 nov.2015]. 
30 Rover, T. Edição de enunciados pelo CNJ divide opiniões de advogados e magistrados. Disponível em http://www.conjur.com.br/2014-jun-25/enunciados-cnj-dividem-opinioesadvogados-magistrados. [Acesso em 16 nov. 2015].

Recebido em: 15/2/2016 Reapresentado em: 29/6/2016 Aprovado em: 8/10/2016

\section{Como citar este artigo:}

Aguiar JR, Santos AO. O conceito de integralidade em saúde nos artigos científicos, no Supremo Tribunal Federal (STF) e nos enunciados do Conselho Nacional de Justiça (CNJ). Revista Cadernos IberoAmericanos de Direito Sanitário. 2016 out./dez, 5(4):96-111. 